\title{
Immune System and Pituitary Tumors: TILs Death tears them Apart. A Review
}

Emigdio José León-Toirac ${ }^{1}$, Erick Robles Torres $^{2}$ and Irma Vega García ${ }^{3}$

${ }^{1}$ Department of Immunology, National Institute of Endocrinology, Havana, Cuba

${ }^{2}$ National Institute of Endocrinology, Havana, Cuba

3Higher Institute of Medical Sciences "Victoria de Girón" Faculty, Havana, Cuba

"Corresponding author: León-Toirac, EJ, Department of Immunology, National Institute of Endocrinology, Zapata esq. D. Revolution Square, Havana, Cuba, Tel: 5352420474; E-mail: emigdio.leon@infomed.sld.cu

Rec date: Apr 06, 2015; Acc date: May 28, 2015; Pub date: May 30, 2015

Copyright: (c) 2015 León-Toirac EJ, et al. This is an open-access article distributed under the terms of the Creative Commons Attribution License, which permits unrestricted use, distribution, and reproduction in any medium, provided the original author and source are credited.

\section{Abstract}

Introduction: Pituitary tumors are common in general population, but the study of these tumors confronts some restrictions, including the inaccessibility of the gland for biopsy material. It has also been described that lymphocytic infiltration can be found adjacent to pituitary adenomas.

Objective: This article was conceived to collect some information to describe the involvement of immune system on pituitary tumors microenvironment and also to shed light to molecular mechanisms implicated on the pathogenesis of these diseases.

Methods: A search of the literature was done in Medline database between 1990 and 2014, using the search terms immune system and pituitary tumors. A review of 88 papers was performed including articles published mainly in English-language and with free access to full paper. The most recent references were used whenever possible.

Results: Cell-mediated immunity appears to be predictive of a less favorable clinical outcome. Also sera antipituitary antibodies can be obtained from patients with those tumors. Low sensitivity of individual autoantibodies makes them, at present, a poor diagnostic marker for clinical purposes.

Conclusions: Nonetheless, tumors infiltrating lymphocytes (TILs) and anti-pituitary antibodies (APA) still make part of a promising calling for diagnosis and treatment of pituitary tumors; new approaches to further understanding of immune response enrollment on pituitary tumor microenvironment are still needed.

Keywords: Anti-pituitary antibodies; Immune system; Pituitary adenomas; Pituitary tumors; Tumor infiltrating lymphocytes

\begin{abstract}
Abbreviations:
APA: Anti-Pituitary Antibodies; AITD: Autoimmune Thyroid Disease; CD: Cluster of Differentiation; CNS: Central Nervous System; CRH: Corticotropin-Releasing Hormone; CDK: Cyclin-Dependent Kinase; ELISA: Enzyme Linked Immunosorbent Assay; Foxp3: Forhead Box p3; HPA: Hypothalamic-Pituitary-Adrenal; IL: Interleukin; MRI: Magnetic Resonance Imaging; NK: Natural Killer; PTTG-1: Pituitary Tumor Transforming Gene 1; PRL: Prolactin; SP: Substance P; Th: T Helper Lymphocyte; TAAs: Tumor-Associated Antigens; TILs: Tumor Infiltrating Lymphocytes; TNF: Tumor Necrosis Factor; MHC: Major Histocompatibility Complex
\end{abstract}

\section{Introduction}

During the last 20 years a tremendous improvement in the care of patients with hypopituitarism and particularly pituitary tumors has been achieved [1,2]. Since neuroimaging techniques have recently improved, pituitary tumors are more frequently diagnosed incidentally [3].
Pituitary tumors are common in general population (16.7\%), Usually, pituitary tumors are assigned as primary tumors of the central nervous system (CNS) and contribute to $5 \%$ to $20 \%$ of all primary CNS tumors. In $95 \%$ of cases, pituitary tumors occur sporadically $[3,4]$. These endocrine tumors are invariably benign adenomas arising from differentiated hormone-expressing cells $[4,5]$.

The study of human pituitary tumors pathogenesis has been challenged by several limitations including the inaccessibility of the gland for biopsy material, lack of functional hormone-secreting human pituitary cell lines and few faithful animal models that recapitulate hormone-secreting or nonfunctional human pituitary tumor phenotypes [5].

In several cases, a slight grade of lymphocytic infiltration can be found adjacent to pituitary adenomas. This infiltration is recognized as tumor infiltrating lymphocytes (TILs) specifics for these endocrinopathies. Infiltration patterns are found as perivascular, interstitial or mixed and almost entirely composed of $\mathrm{T}$ cells $[6,7]$. Also anti-pituitary antibodies (APA) can be obtained from sera of patients with pituitary tumors. Whether the presence of APA is integrated with TILs response in pituitary adenoma and which is their clinical significance in this setting is currently unknown [6].

This article was conceived to collect some information to describe the involvement of immune system on pituitary tumors 
microenvironment and also to shed light to molecular mechanisms implicated on the pathogenesis of these diseases.

\section{Review Criteria}

A Medline database search was conducted to identify articles published between 1990 and 2014 that focused on immune system and pituitary tumors. Search terms used were: "pituitary tumors", "pituitary adenomas" in association with "immune system", "immunity", "tumor infiltrating lymphocytes", "anti-pituitary antibodies" and "autoimmunity". The majority of papers selected were English-language, full-text papers (88 articles) in order to scrutiny particular aspects on this topic. We also searched the reference lists of identified review articles for further primary information. The most recent references were used whenever possible.

\section{Integrative Perspective of Pituitary Gland}

The pituitary gland is critically important for regulating growth, reproduction and the maintenance of homeostatic mechanisms. Much of this control comes from the anterior pituitary gland, which secretes polypeptide hormones from five specialized cell types. Disruption of the synthesis and secretion of one or more of these hormones can result in hormone deficiency diseases $[5,8]$.

Complex control of anterior pituitary function involves hypothalamic, intrapituitary and peripheral signals. Pituitary cell integration of these three tiers of control results in coordinated regulation of differentiated pituitary hormone synthesis and secretion, as well as selective regulation of pituitary cell proliferation [5].

Rhythmic synchronization of the body occurs indirectly through behavioural rest-activity cycles but also in a direct manner through a complex and incompletely understood interplay between hormones and local autonomic innervations [9,10]. The key hormones implicated in this process - glucocorticoids and catecholamines (specifically, adrenaline and noradrenaline) - are released by the adrenal gland via the hypothalamic-pituitary-adrenal axis (HPA axis) $[11,12]$.Glucocorticoids can inhibit the synthesis of pro-inflammatory factors, such as cytokines interleukin $1 \beta$ (IL1 $\beta$ ), interleukin 6 (IL6) and tumor necrosis factor (TNF) [12]; and promote the expression of antiinflammatory mediators. Catecholamines can have diverse effects on the immune system, including the increase of numbers of neutrophils and natural killer (NK) cells in the blood, and boosting humoral immune responses $[9,13]$.

Investigating the molecular biology of pituitary development has allowed translation of research findings into the clinic and subsequent reciprocal transfer of information back from patients to the basic science laboratory [8].

Some research findings [14] suggest that high-locomotion rodent females experience life-long elevations in glucocorticoids responses to novelty, and that these elevated levels may be comparable to chronic stress. CNS perceptions of threat from environmental stressors, particularly when experienced chronically, lead to downstream activation of neuroendocrine pathways including the autonomic nervous system and the HPA axis. This naturally-occurring endocrine profile may influence immune responses which in turn could affect disease susceptibility $[11,12,15,16]$.

Increasing evidence implicates mast cells in inflammatory diseases, especially those exacerbated by stress [11,17]. Mast cells are ubiquitous in the human body and are critical for allergic reactions, but are also involved in innate and acquired immunity during which they secrete numerous vasoactive molecules, cytokines and proteases. Corticotropin-releasing hormone $(\mathrm{CRH})$ is secreted under stress and activates the HPA axis $[9,11]$. Human mast cells express receptors for $\mathrm{CRH}$ and other neuropeptides such as substance P (SP). Mast cell stimulation by SP and $\mathrm{CRH}$, would result in secretion of proinflammatory cytokines that would recruit and activate local immune accessory cells that would also stimulate peripheral nerves. All this interaction makes part of a local molecular network mimicking the HPA axis [17].

\section{Featuring Pituitary Tumors}

Pituitary tumors constitute up to $15 \%$ of intracranial neoplasms $[5,18,19]$, and have an overall population prevalence of $\sim 80-90$ per 100,000 of the population. These adenomas secrete specific hormones reflective of their differentiated cell of origin [3].

Pituitary adenomas are classified partially depending on their size into microadenomas $(<1 \mathrm{~cm})$ and macroadenomas $(>1 \mathrm{~cm})[3,18]$. The size of the adenoma corresponds with compromising effects on the optic chiasm, cranial nerves, and cavernous sinuses, but tumor size does not reflect its clinical importance. This classification is supplemented by immunochemistry and functional status $[3,20]$.

Pituitary tumors are also classified as functioning or nonfunctioning on the basis of their ability to produce and secrete mature hormones. Approximately half to one third of all pituitary tumors are non-functioning pituitary adenomas (i.e. without hormone secretion detected either by immunohistochemistry or by elevated hormonal blood levels) $[3,20]$. The most common hormone-secreting pituitary tumor is the prolactinoma (lactotroph adenoma, $25 \%-41 \%$ ) $[3,18,21]$, followed by somatotroph adenomas (10\%-15\%), corticotroph adenomas (about 10\%) [3,19].

Gonadotroph cells can also give rise to tumors but few secrete intact luteinizing hormone or follicle-stimulating hormone and most are nonfunctioning. These gonadotroph-derived tumors are usually discovered incidentally, or patients may present with pituitary failure or local central features of mass expansion [5].

Pituitary tumors are mostly monoclonal benign adenomas [3,5], rather than hyperplastic growths, which suggests that they in fact arise from expansion of single precursor cells that possess a unique proliferative advantage. Several lines of evidence point to a source of pituitary progenitor cells that may give rise to pituitary adenomas [5]. Pituitary carcinomas are extremely rare, and in these cases the assumption is that they are former benign adenomas that have undergone additional genetic mutations [3].

Specific tumor-initiating and tumor-promoting factors have been characterized in animal models, as well as in limited human tissue samples. These factors have been shown to confer increased proliferative potential to the precursor cell for adenoma formation and subsequent tumor growth, and include genetic or epigenetic abnormalities, paracrine growth factor disruptions and an altered intrapituitary microenvironment. The primary initiating cause for these events remains elusive, although several transgenic mouse models have demonstrated that either inactivated or over expressed cell cycle regulators are sufficient to initiate pituitary tumorigénesis $[4,5,8,22]$.

INK4 and CIP/KIP are two distinct families of cyclin-dependent kinase $(\mathrm{CDK})$ inhibitors implicated in mediating a wide range of cell 
growth control signals [5]. In an experimental model [23], p18(INK4c)-deficient mice develop gigantism and widespread organomegaly. The pituitary gland, spleen, and thymus were disproportionately enlarged and hyperplastic. $\mathrm{T}$ and $\mathrm{B}$ lymphocytes developed normally in p18-deficient mice, but both exhibit increased cellularity and a higher proliferative rate upon mitogenic stimulation. Moreover, disruption of the CDK inhibitor genes p27 in mice results in a series of additional novel phenotypes including increased body size, multiorgan hyperplasia, female sterility, retinal dysplasia, and pituitary tumors $[5,24]$.

\section{Tumors met Immune System}

Tumors are frequently infiltrated by lymphocytes. Whereas it is established that the immune system has the capacity to recognize tumor antigens, it remains unclear whether it protects the host from tumor growth and spread. In some tumors, like germinomas, melanoma, ovarian, breast, and papillary thyroid cancer, TILs are often numerous; in other tumors, TILs are scanty or have not been systematically characterized $[6,25]$.

TILs have, in fact, several phenotypes. Effector CD8 lymphocytes and NK cells are beneficial to the host because they directly contact the tumor cell and initiate a cytotoxic cascade that eventually kills the tumor cells. They also contribute to the antitumor response by an antibody-dependent target cell killing. On the contrary, suppressor (or regulatory) TILs are detrimental for the patient. They are characterized by the expression of CD4, CD25, and Foxp3; and inhibit effector lymphocytes in a cytokine or cell-contact dependent fashion, ultimately dampening their beneficial antitumor activities [6,26].

TILs and other mononuclear cells, like tumor-associated macrophages and dendritic cells, are recruited to the tumor site through several mechanisms including specific recognition of tumor antigens, aspecific inflammatory response to necrotic tumor cells, and chemotaxis [6]. Pro-inflammatory cytokines and/or antigenic fragments released from tumoral tissue could provoke a diffuse inflammatory reaction within the tumor [7].

Actually, the immune system is capable of discriminating between benign and malignant cells by recognizing aberrantly expressed proteins/peptides exposed on the cell surface in the context of the major histocompatibility complex (MHC). Immune responses are also highly dependent on the tumor's microenvironment [27], and it is generally characterized by cellular immunity, including NK cell cytotoxicity and T-cell production of cytokines [16].

However, tumors have well-developed escape mechanisms by which they interfere with immune cell signaling and thus evade recognition and destruction by the immune response. Thus the immune response in the tumor microenvironment is substantially down-regulated compared to that in peripheral blood. Down-regulation of the immune response induced by neuroendocrine stress hormones is a potential mediator of impaired surveillance in tumor microenvironment. Hence it asserts a systemic influence on tumor growth and metastatic spread [15].

Biobehavioral factors are related, for instance, to immune activity in the tumor microenvironment, specifically the cellular immune response in TILs. Social support is related to greater NK cell activity in both peripheral blood and TILs, whereas distress is associated with blunted NK cell activity in TILs and poorer T-cell production of T helper 1 against T helper 2 (Th1 vs.Th2) cytokines in peripheral blood, ascites, and TILs [15,16,28].

The phenotype and functional consequences of TILs are complex. Not surprisingly, the prognostic relevance of tumor autoimmunity, defined here as the presence of TILs or antibodies directed against tumor antigens, remains to be elucidated for most human cancer $[6,25,29]$.

\section{Pituitary Tumors and Immune System: Marriage in- explored}

Very limited information is available for TILs present in pituitary adenomas. A slight grade of lymphocytic infiltration, also referred to as secondary hypophysitis, can be found adjacent to pituitary adenomas as well as in other tumors such as Rathke's cleft cyst and craniopharyngioma and is considered to be a peritumoral inflammatory reaction $[6,29]$.

A different condition seems to be the case of lymphocytic infiltration within the adenomatous lesion. TILs have been described to be present within the adenoma lesion in 3\% of a cohort of 1400 secreting and nonsecreting adenomas. This study [6] showed that the presence of TILs influences the disease progression independently from the adenoma size and type of hormonal secretion. In addition, this data supports well the hypothesis that pituitary adenomas act as other tumors in predisposing to cell-mediated autoimmunity development, showing pituitary adenomas as an intermediate condition between normal pituitary gland and hypophysitis.

Autoantibodies are a classic marker of autoimmune diseases and are of great help for the diagnosis, prediction and, to some extent, monitoring of the remission of the disease. It comes into view that tumor immunology is on the way to developing similar tools that will be valuable in the serological identification of tumors. Interestingly, detecting cancer antigen-related autoantibodies has proven useful in early diagnosis of certain malignant tumors and may precede the clinical manifestation of the tumor $[6,30]$.

In a fine study [31], an analysis of the peripheral lymphocyte population revealed that patients with prolactinoma had a higher percentage of $B$ cells than normal subjects, while there was no significant difference in the percentages of total $\mathrm{T}$ lymphocytes or in the helper and suppressor $\mathrm{T}$ cell ratios in the two groups of subjects. These results suggest that prolactin (PRL) regulates humoral immune responses in man directly by stimulating B lymphocytes or indirectly by inhibiting suppressor T lymphocyte activity. B lymphocytes are the main cells implicated in an antibody mediated immune response.

Autoantibodies to human pituitary cytosol proteins were determined by immunoblotting in sera from patients with hypopituitarism and their relatives. Reactivity to an $\mathrm{M}(\mathrm{r}) 49,000$ protein was significantly more frequent in patients (6/21 (28\%) $\mathrm{P}<0.05)$ as well as in relatives $(10 / 35(28 \%) \mathrm{P}<0.02)$ compared with controls $(3 / 44(6.8 \%))$. APA to this particular protein has previously been detected in sera from $70 \%$ of patients with biopsy-proven lymphocytic hypophysitis [32]. Lymphocytic hypophysitis is characterized by diffuse infiltration of the pituitary by a mixed population of chronic inflammatory cells, with oncocytic change of parenchymal cells, and variable amounts of fibrosis; so, is widely suspected to be an autoimmune phenomenon [29,33-35], because its epidemiological, histomorphological and clinical features are suggestive for an autoimmune pathogenesis [35]. Since organ specific 
autoantibodies are frequent in patients with autoimmune endocrine disease as well as in their unaffected relatives, autoantibodies to this $M(r)$ 49,000 pituitary cytosolic protein may represent markers for an immunological process affecting the pituitary gland [32].

Serum APA have been measured so far in pituitary adenoma patients using different techniques (mainly immunofluorescence, immunoblotting, ELISA, and radioligand assay) and were found to have a prevalence ranging between 0 and 30\% [6,36,37]. Even more, a high prevalence of APA-positivity has been detected in patients with autoimmune thyroid disease (AITD), particularly those with Hashimoto's thyroiditis. The presence of APA, also correlated with an increased incidence of growth hormone deficiency, suggests that patients with AITD should be routinely monitored for pituitary dysfunction [33,38-40].

However, the overall low sensitivity of individual autoantibodies makes them, at present, a poor diagnostic marker for clinical purposes. Limitation/efficacy of immunological markers primarily relies on the antigen specificity of the methods used, and therefore, APA cannot, at present, be used in clinical practice [6,39].

The original APA assays are now regarded as nonspecific, and their use is restricted by limited access. In vitro transcription and translation assays offer the prospect of more-specific antigen detection, and $\alpha$-enolase and tudor-domain containing protein 6 have emerged as novel pituitary autoantigens $[4,33]$.

\section{From New Perspectives}

Nowadays, the optimum treatment option (surgery, medical therapy, radiotherapy) very much depends upon the individual type of pituitary tumor [41]. Even when mortality after surgery of pituitary adenomas has been shown to be dependent on many preoperative risk factors, peroperative major complications may exist [1].

Improved understanding of the signaling pathways that regulate pituitary tumorigenesis in the past few years has led to substantial progress in the development of new compounds for the treatment of pituitary tumors $[27,41,42]$.

For instance, it has been shown that the addition of the systemic cytokine Interleukin 2 (IL-2) as a tumor immunization therapy plays a pivotal role in increasing the frequency of immune cancer rejections $[27,43]$.

Pituitary tumor transforming gene 1 (PTTG-1), a securin proangiogenic pituitary factor, has shown to be associated with tumorigenesis, angiogenesis and cancer progression [5,27]. PTTG-1 is over expressed in pituitary tumors, thyroid cancer, and so many other. This molecular finding could be an important point of departure in the quest for a therapeutic target.

An experience of successful immunotherapy to treat a pituitary macroprolactinoma was reported years ago as a promising strategy for the treatment of these tumors [42]. A Th1 activator adjuvant was inoculated with autoantigens intradermally once per week for 24 consecutive weeks. Th1 cytokine network induces activation of both nonspecific (NK cells) and specific (cytotoxic $\mathrm{T}$ lymphocytes) immunity. After immunotherapy, serum PRL concentration decreased, the patient's symptoms disappeared, and a follow-up brain magnetic resonance imaging (MRI) revealed almost complete disappearance of the tumor.
Such an application of cell-mediated autoimmunity on pituitary tumor microenvironment was revealed by a pioneering study [44], on which GH3 pituitary adenoma cells were implanted subcutaneously into elder rats with hypoplasic thymus, showing that it is possible to regenerate normal thymic tissue in situ and reverse the natural loss in cell-mediated immunity that occurs with aging [27].

Tapping into the process of immune surveillance through the identification and evaluation of autoantibodies against tumorassociated antigens (TAAs) represents a promising avenue of biomarker development. Such trends provide a basis for the discernment of the specific challenges currently facing autoantibody biomarker development, and lay the groundwork for future innovations aimed at overcoming that challenges [45].

Discovering novel TAAs is only the first step in improving the complementary use of two biotherapic approaches (active immunization/adoptive transfer of tumor antigen-specific $\mathrm{T}$ cells) and to better design future simple and safe clinical studies $[27,46,47]$.

\section{Conclusion}

Identifying immune response components against TAA has become a formidable task for tumor immunologists. TILs and APA, present in a significant number of pituitary adenoma patients, make part of this promising calling.

Because of cell-mediated immunity seems to be predictive of a less favorable clinical outcome, autoimmune hypophysitis must emerge as one of the more common diagnoses in clinical endocrinology.

Moreover, novel hypotheses for basic and clinical sciences approaches to further understanding of immune response enrollment on pituitary tumor microenvironment are still needed.

\section{Declaration of Interest}

The authors declare that there is no conflict of interest that could be perceived as prejudicing the impartiality of the research reported.

\section{Authors' Contributions}

EJLT carried out the bibliographic research contributing with main ideas to conceive the study and fully participate in the sequence alignment and draft of the manuscript. ER contributed to conceive the study and helped to draft the manuscript. IV participated in the sequence alignment of the manuscript. All authors read and approved the final manuscript.

\section{References}

1. Erfurth EM, Siesjö P, Björk-Eriksson T (2013) Pituitary disease mortality: is it fiction? Pituitary 16: 402-412.

2. Romero CJ, Nesi-França S, Radovick S (2009) The molecular basis of hypopituitarism. Trends Endocrinol Metab 20: 506-516.

3. Kopczak A, Renner U, Karl Stalla G (2014) Advances in understanding pituitary tumors. F1000Prime Rep 6: 5.

4. Jiang X, Zhang X (2013) The molecular pathogenesis of pituitary adenomas: an update. Endocrinol Metab (Seoul) 28: 245-254.

5. Melmed S (2011) Pathogenesis of pituitary tumors. Nat Rev Endocrinol 7: 257-266.

6. Lupi I, Manetti L, Caturegli P, Menicagli M, Cosottini M, et al. (2010) Tumor infiltrating lymphocytes but not serum pituitary antibodies are 
associated with poor clinical outcome after surgery in patients with pituitary adenoma. J Clin Endocrinol Metab 95: 289-296.

7. Cakir E, Bayindir C, Sabanci PA, Imer M, Ozbey NC (2011) Pituitary macroadenoma with persisting dense lymphocytic infiltration in a young male patient. Clin Neuropathol 30: 318-323.

8. Prince KL, Walvoord EC, Rhodes SJ (2011) The role of homeodomain transcription factors in heritable pituitary disease. Nat Rev Endocrinol 7: 727-737.

9. Scheiermann C, Kunisaki Y, Frenette PS (2013) Circadian control of the immune system. Nat Rev Immunol 13: 190-198.

10. Dickmeis T (2009) Glucocorticoids and the circadian clock. J Endocrinol 200: 3-22.

11. Chrousos GP (2009) Stress and disorders of the stress system. Nat Rev Endocrinol 5: 374-381.

12. Tracey KJ (2009) Reflex control of immunity. Nat Rev Immunol 9: 418-428.

13. O'Neill JS, Reddy AB (2011) Circadian clocks in human red blood cells. Nature 469: 498-503.

14. Cavigelli SA, Bennett JM, Michael KC, Klein LC (2008) Female temperament, tumor development and life span: relation to glucocorticoid and tumor necrosis factor alpha levels in rats. Brain Behav Immun 22: 727-735.

15. Lutgendorf SK, Sood AK (2011) Biobehavioral factors and cancer progression: physiological pathways and mechanisms. Psychosom Med 73: 724-730.

16. Costanzo ES, Sood AK, Lutgendorf SK (2011) Biobehavioral influences on cancer progression. Immunology and Allergy Clinics of North America 31: 109-132.

17. Asadi S, Alysandratos KD, Angelidou A, Miniati A, Sismanopoulos N, et al. (2012) Substance P (SP) induces expression of functional corticotropin-releasing hormone receptor- (CRHR-1) in human mast cells. J Invest Dermatol 132: 324-329.

18. Bronstein MD, Paraiba DB, Jallad RS (2011) Management of pituitary tumors in pregnancy. Nat Rev Endocrinol 7: 301-310.

19. Yamada M, Mori M (2008) Mechanisms related to the pathophysiology and management of central hypothyroidism. Nat Clin Pract Endocrinol Metab 4: 683-694.

20. Gonzales PH, Mezzomo LC, Ferreira NP, Roehe AV, Kohek MB, et al (2015) Aromatase P450 expression in human pituitary adenomas. Neuropathology 35: 16-23.

21. Klibanski A (2010) Clinical practice. Prolactinomas. N Engl J Med 362: 1219-1226.

22. Armaiz-Pena GN, Cole SW, Lutgendorf SK, Sood AK (2013) Neuroendocrine influences on cancer progression. Brain Behav Immun 30 Suppl: S19-25.

23. Franklin DS, Godfrey VL, Lee H, Kovalev GI, Schoonhoven R, et al (1998) CDK inhibitors p18(INK4c) and p27(Kip1) mediate two separate pathways to collaboratively suppress pituitary tumorigenesis. Genes Dev 12: 2899-2911.

24. Bellodi C, Krasnykh O, Haynes N, Theodoropoulou M, Peng G, et al. (2010) Loss of function of the tumor suppressor DKC1 perturbs p27 translation control and contributes to pituitary tumorigenesis. Cancer Res 70: 6026-6035.

25. Uppaluri R, Dunn GP, Lewis JS Jr (2008) Focus on TILs: prognostic significance of tumor infiltrating lymphocytes in head and neck cancers. Cancer Immun 8: 16.

26. Kosmaczewska A, Ciszak L, Potoczek S, Frydecka I (2008) The significance of Treg cells in defective tumor immunity. Arch Immunol Ther Exp (Warsz) 56: 181-191.

27. Chiriva-Internati M, Ferrari R, Prabhakar M, Yu Y, Baggoni L, et al. (2008) The pituitary tumor transforming gene (PTTG-1): an immunological target for multiple myeloma. J Transl Med 6: 15.
28. Green McDonald P, O'Connell M, Lutgendorf SK (2013) Psychoneuroimmunology and cancer: a decade of discovery, paradigm shifts, and methodological innovations. Brain Behav Immun 30 Suppl: S1-9.

29. Gutenberg A, Bell JJ, Lupi I, Tzou SC, Landek-Salgado MA, et al. (2011) Pituitary and systemic autoimmunity in a case of intrasellar germinoma. Pituitary 14: 388-394.

30. Chanson P, Salenave S (2004) Diagnosis and treatment of pituitary adenomas. Minerva Endocrinol 29: 241-275.

31. Ishibashi M, Kuzuya N, Sawada S, Kitamura K, Kamoi K, et al. (1991) Anti-thyroid antibodies in patients with hyperprolactinemia. Endocrinol Jpn 38: 517-522.

32. Strömberg S, Crock P, Lernmark A, Hulting AL (1998) Pituitary autoantibodies in patients with hypopituitarism and their relatives. J Endocrinol 157: 475-480.

33. Ezzat S (2008) Epigenetic control in pituitary tumors. Endocr J 55: 951-957.

34. Hamnvik OP, Laury AR, Laws ER Jr, Kaiser UB (2010) Lymphocytic hypophysitis with diabetes insipidus in a young man. Nat Rev Endocrinol 6: 464-470.

35. Falorni A, Minarelli V, Bartoloni E, Alunno A, Gerli R (2014) Diagnosis and classification of autoimmune hypophysitis. Autoimmun Rev 13: 412-416.

36. Manoranjan B, Salehi F, Scheithauer BW, Rotondo F, Kovacs K, et al. (2010) Estrogen receptors alpha and beta immunohistochemical expression: clinicopathological correlations in pituitary adenomas. Anticancer Res 30: 2897-2904.

37. Moya Chimenti E, Alvarez Doforno R, Villaroel Bajo A, Frutos R Pallardo Sánchez LF, et al. (2010) [Antipituitary antibodies in patients with suspected autoimmune hypophysitis]. Endocrinol Nutr 57: 160-164.

38. Manetti L, Lupi I, Morselli LL, Albertini S, Cosottini M, et al. (2007) Prevalence and functional significance of antipituitary antibodies in patients with autoimmune and non-autoimmune thyroid diseases. J Clin Endocrinol Metab 92: 2176-2181.

39. Shelly S, Boaz M, Orbach H (2012) Prolactin and autoimmunity. Autoimmun Rev 11: A465-470.

40. Boi F, Minerba L, Lai ML, Marziani B, Figus B, et al. (2013) Both thyroid autoimmunity and increased serum TSH are independent risk factors for malignancy in patients with thyroid nodules. J Endocrinol Invest 36: 313-320.

41. Gueorguiev M, Grossman AB (2011) Pituitary tumors in 2010: a new therapeutic era for pituitary tumors. Nat Rev Endocrinol 7: 71-73.

42. Hazrati SM, Aghazadeh J, Mohtarami F, Abouzari M, Rashidi A (2006) Immunotherapy of prolactinoma with a $\mathrm{T}$ helper 1 activator adjuvant and autoantigens: a case report. Neuroimmunomodulation 13: 205-208.

43. Sim GC, Radvanyi L2 (2014) The IL-2 cytokine family in cancer immunotherapy. Cytokine Growth Factor Rev 25: 377-390.

44. Kelley KW, Brief S, Westly HJ, Novakofski J, Bechtel PJ, et al. (1986) GH3 pituitary adenoma cells can reverse thymic aging in rats. Proc Natl Acad Sci U S A 83: 5663-5667.

45. Nolen BM, Lokshin AE (2010) Autoantibodies for cancer detection: still cause for excitement? Cancer Biomark 6: 229-245.

46. Zhu Q, Liu M, Dai L, Ying X, Ye H, et al. (2013) Using immunoproteomics to identify tumor-associated antigens (TAAs) as biomarkers in cancer immunodiagnosis. Autoimmun Rev 12: 1123-1128.

47. Liu W, Peng B, Lu Y, Xu W, Qian W, et al. (2011) Autoantibodies to tumor-associated antigens as biomarkers in cancer immunodiagnosis. Autoimmun Rev 10: 331-335. 